\title{
Condolence and Empathy in Online Communities
}

\author{
Naitian Zhou \\ Computer Science and Engineering \\ University of Michigan \\ naitian@umich.edu
}

\author{
David Jurgens \\ School of Information \\ University of Michigan \\ jurgenseumich. edu
}

\begin{abstract}
Offering condolence is a natural reaction to hearing someone's distress. Individuals frequently express distress in social media, where some communities can provide support. However, not all condolence is equal-trite responses offer little actual support despite their good intentions. Here, we develop computational tools to create a massive dataset of $11.4 \mathrm{M}$ expressions of distress and $2.8 \mathrm{M}$ corresponding offerings of condolence in order to examine the dynamics of condolence online. Our study reveals widespread disparity in what types of distress receive supportive condolence rather than just engagement. Building on studies from social psychology, we analyze the language of condolence and develop a new dataset for quantifying the empathy in a condolence using appraisal theory. Finally, we demonstrate that the features of condolence individuals find most helpful online differ substantially in their features from those seen in interpersonal settings.
\end{abstract}

\section{Introduction}

Millions of individuals experience emotional distress each year from diverse circumstances such as personal loss or abuse. After such experiences, people often turn to their social circle in social media to convey their experiences and seek out emotional support (Brubaker et al., 2012; Brubaker and Hayes, 2011; De Choudhury and Kiciman, 2017). Often, support comes in the form of condolence where individuals connect with the distressed person, and express forms of sympathy, empathy, advice, and social connection, among others (Burleson, 2003). However, not all expressions of distress receive emotional support, nor do all condolence messages offer equal levels of support (Davidowitz and Myrick, 1984). Given the wide-spread use of social media for seeking social support, what makes for an effective supportive message? Here, we perform the first major study of condolence in social media, examining what type of distress individuals seek support for, what linguistics factors are more likely to elicit condolence, and what types of condolence viewed as more helpful.

Distress and emotional support have long been explored in work in social psychology and counseling (Burleson et al., 2009; Rack et al., 2008), frequently around bereavement and helping victims of abuse. NLP works have only recently examined emotional support in online spaces for mental and physical health (Biyani et al., 2014; Navindgi et al., 2016; Wang et al., 2015) and in communities oriented around goals like weight-loss (Manikonda et al., 2014); however, these focus on the general concept of supportiveness. In this work, we examine distress as a universal phenomenon-not just related to health and death - and examine the strategies and helpfulness of responses to this distress.

This study aims to computationally identify mechanisms and strategies for delivering effective and impactful condolence on social media. Conveying condolence is often difficult for many people (Cameron et al., 2019), who fall back to common responses to distress such as "thoughts and prayers" or "I'm so sorry for your loss" due to the emotional and mental effort required to relate to the distressed person. To identify effective strategies of condolence, we construct a dataset of $14.1 \mathrm{M}$ expressions of distress from Reddit by developing computational models for recognizing distress and condolence. We then use this dataset to analyze how the community embraces the individual and which condolence responses were found helpful.

This work offers the following three contributions. First, we introduce a new massive dataset of $11.4 \mathrm{M}$ public expressions of distress and $2.8 \mathrm{M}$ of condolence labeled using two deep learning models for identifying each, showing that our data mirrors known trends in seasonality and theme. Sec- 
ond, using an analysis of $11.4 \mathrm{M}$ expressions of distress, we demonstrate that the community selectively engages in condolence; not all distress messages which attract attention actually receive support. Third, we introduce a new dataset and model for identifying empathy in condolences and, using the empathy estimates, find that distressed individuals less frequently offer gratitude for deeply empathetic condolences and instead prefer compassionate, positive messages, which runs counter to observations from in-person settings.

\section{Recognizing Distress and Condolence}

Distress and condolence are expressed in a variety of ways. As no standard dataset exists for detecting these constructs, we first create one for training models using distant supervision to heuristically label data. Then, two classifiers are trained to recognize each in expressions on social media and finally fine-tuned to attain high precision. For both, we use Reddit comments as our base data. Additional details for classification and training are reported in supplemental section A.

Recognizing Distress A set of stereotypical condolence expressions, e.g., "sorry for your loss" or "my heart goes out to you" is first manually identified. Due to their ubiquitous use in the face of distress, such expressions act as heuristics to identify posts containing a variety of circumstances and topics. All Reddit comments receiving at least one of these stereotypical-condolence replies are treated as positive examples of distress, identified from all Reddit comments in the year 2017. ${ }^{1}$ An equivalent number of randomly-selected comments that do not receive any of these stereotyped-condolence responses are sampled from the same communities in the same month, which ensures the corpus is topically and temporally balanced. In total, 229,204 comments are collected as training data from Reddit during the year 2017.

Two classifiers are trained from this balanced dataset. The first is a SVM classifier using unigrams and bigrams, which is known to be a robust baseline (Wang and Manning, 2012). The second is a BERT-based classifier (Devlin et al., 2019) trained using a linear layer on top of the pooled [CLS] token for classification over 2 epochs. The base pretrained model was

\footnotetext{
${ }^{1}$ No filtering was done to pre-select only those posts that might elicit distress-like comments that might receive such condolences.
}

\begin{tabular}{|c|c|c|c|c|}
\hline & & Precision & Recall & F1 Score \\
\hline \multirow{3}{*}{ 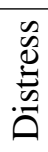 } & Random & 0.5 & 0.5 & 0.5 \\
\hline & SVM & 0.597 & 0.631 & 0.617 \\
\hline & BERT & 0.725 & 0.686 & 0.705 \\
\hline \multirow{3}{*}{$\begin{array}{l}\dot{0} \\
\dot{0} \\
\dot{0}\end{array}$} & Random & 0.5 & 0.5 & 0.5 \\
\hline & SVM & 0.745 & 0.897 & 0.815 \\
\hline & BERT & 0.908 & 0.767 & 0.831 \\
\hline
\end{tabular}

Table 1: Model performances at recognizing expressions of distress (top) and condolence (bottom) from the heuristically-labeled data.

bert-base-uncased from the Hugging Face transformers library (Wolf et al., 2019). For both models, comments are preprocessed to remove markdown, links, and non-ASCII characters. Table 1 (top) shows that models are able to accurately identify distress expressions. Because many contexts can elicit an emotional response, distress is challenging to identify; further, because the data is heuristically labeled, we do not expect high performance in this initial model.

Recognizing Condolence Condolence-giving comments are heuristically identified in a similar manner to those for distress. When a comment receives a reply containing one of the stereotypedcondolence expressions, a single different reply to that same comment is selected as another expression of condolence. The assumption is that distress attracts multiple condolences, allowing us to learn a variety of condolence expressions. To minimize potential confounds, condolence comments were collected from all Reddit comments from a different year than distress comments (2016). Negative examples of condolence are randomly sampled from replies to different non-distress comments under the same post, which ensures a balance in time and subreddit between positive and negative examples.

SVM and BERT-based classifiers were tested to recognize condolence in comments, using the same setup as those for recognizing distress. Performance at recognizing condolence, shown in Table 1 (bottom) was even higher than that for recognizing distress. Since there are relatively common strategies in condolence expressions (e.g., expressing sympathy with phrases like "I'm so sorry for your loss"), we suspect these condolence comments are easier to recognize. 
Tuning for Precision Decision thresholds were set at 0.9 for both classifiers to focus on precision after a manual review of a subset of classifications found this to produce sufficiently correct results.

Dataset Description Our final condolence and distress datasets were collected by running the respective classifiers on a random sample of 2018 Reddit comments made in the top ten thousand most popular safe-for-work subreddits. Condolence comments have a length centered around a median of 21 words, with a long right tail (mean of 47.7 words, standard deviation of 79.8 words). Distress comments have a similarly shaped distribution, with a median of 25 words, mean of 41.3 words, and standard deviation of 57.8 words.

\section{Condolence Behavior in Social Media}

As an initial demonstration of the model, we label a random sample of Reddit comments from 2018 made in the top ten thousand most popular safefor-work subreddits and examine where and when distress and condolence are exhibited.

Distress and condolence communities Figure 1 (left) shows that while health topics are prominent, individuals frequently seek out communities based around bereavement (e.g., r/Miscarriage) and abuse (e.g., r/domesticviolence). This result confirms that our model is able to identify a diverse set of circumstances in which individuals experience distress, mirroring some of those highlighted in prior work for online support of distress (Krysinska and Andriessen, 2013; Huh et al., 2014; Döveling, 2017). Surprisingly, the location of condolence behavior (Figure 1, right) does not mirror that of distress. Instead, condolence is frequently offered to those suffering from the loss of a pet and, less frequently, those experiencing the death of a loved one. Many people find the death of a pet more relatable compared with other circumstances like domestic violence, lessening the effort required to relate to the person experiencing the loss and offer condolence (Lim and DeSteno, 2016). Indeed, to express effective condolence, an empathetic response requires effort to relate on a personal level to the feelings of the affected person (Cameron et al., 2019), which many may find more challenging emotionally in circumstances like abuse.

Seasonal effects in distress Changes in seasons and holidays are both known to increase distress and anxiety levels (Cattell, 1955; Rosenthal et al., 1984; Harmatz et al., 2000). As Figure 2
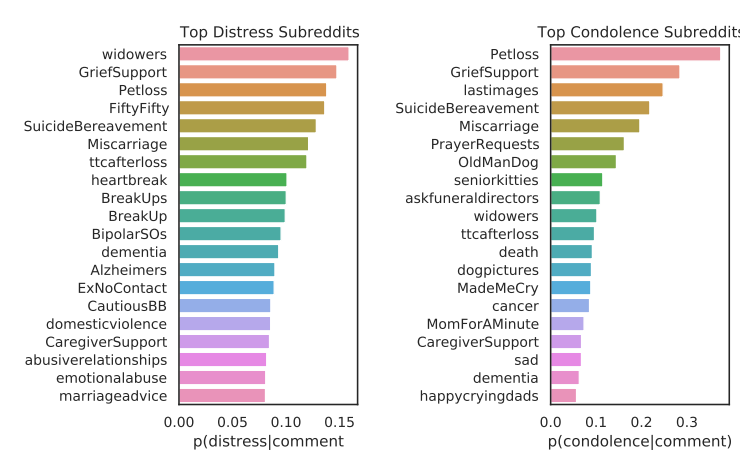

Figure 1: Subreddits with highest proportion of condolence and distress comments.

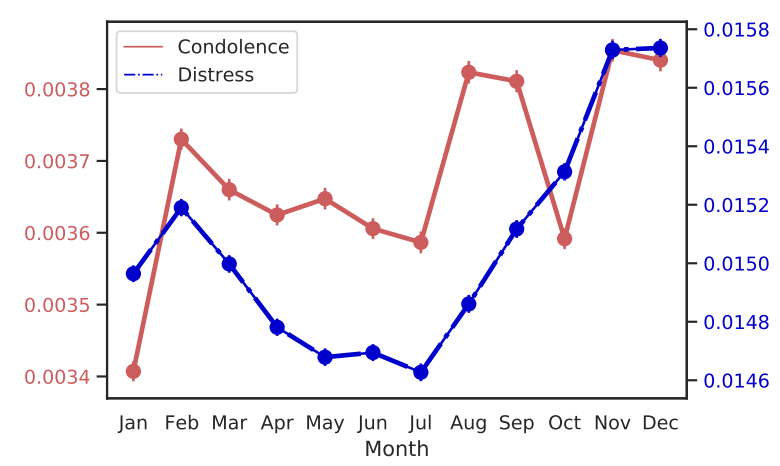

Figure 2: Relative rates for distress (right axis) and condolence (left axis) show that while distress mirrors expected seasonal trends, condolence does not; instead, condolence trends are partially driven by response to events, e.g., mass shootings. Throughout the paper, error bars show $95 \%$ confidence intervals.

shows, expressions of distress in Reddit mirror these trends with a substantial increase around commonly-celebrated holidays. There are spikes around Valentine's Day (February) and increases leading to Thanksgiving (November), Christmas and New Year's (December). Surprisingly, the rate of a community's support of these individualsexpressed through condolence-largely does not mirror this trend. Instead, we observe that spikes in condolence were associated with significant events, including school shootings and celebrity deaths; these self-contained events triggered mass outpourings of condolence.

\section{What Distress Receives Condolence?}

As individuals turn to social platforms for emotional support for a variety of reasons, which types of distress messages receive condolence? We contrast whether an expression of distress receives condolence with receiving any reply.

Methods To understand what factors lead to a dis- 
tress message receiving a reply or a condolence, we fit separate mixed-effect logistic regression models on the dependent variable of receipt of the respective type. To capture thematic trends across messages, we train a 20-topic LDA model and manually label each topic with its prominent theme (topics are shown in supplemental section C). Offering a condolence can require empathetic alignment with another person (Trobst et al., 1994; Cameron et al., 2019), which could be difficult for certain emotions; therefore, we include estimates of the emotions expressed in a distress message using the NRC-emotion lexicon (Mohammad and Turney, 2013). Pronouns reflect the narrative focus of the distress, e.g., frequent mentions of "I" center the content on the distressed person whereas "he" focus on what was done to the distressed person; therefore we include counts of how many times first, second, and third-person pronouns appear using LIWC categories (Pennebaker et al., 2001). Individuals on Reddit are known to be sensitive to the perceived gender of the author when providing support (Wang and Jurgens, 2018), so we include a variable for the user's estimated gender using genderperformr. As controls, we include comment length by space-delimited words, the comment age in hours after the post was created, the depth of the comment, the score of the post as a measure of popularity, and temporal factors for hour of day, day of week, and month. To control for differences within specific subreddits and posts, we include nested random effects for subreddit and the post in which the distress comment is made; for computational tractability, we include only random effects for posts with 30 or more distress comments. The Reddit-based models were fit using a random sample of $1 \mathrm{M}$ comments from the 2018 data identified as distress expressions.

Results The factors affecting whether a distress comment receives a reply differed substantially from those receiving condolence. Whereas distress comments relating to politics, dieting, or sports are likely to receive a reply, such comments are far less likely to receive condolence. Differences in topical effects show that while the Reddit community is likely to engage with distress in all topics, the community selectively supports only a few of these. While the model for receiving a reply is similar to De Choudhury and De (2014, table 8$)$ who examined mental health, these results point to the importance of looking at the content of the replies,

\begin{tabular}{|c|c|c|}
\hline & reply & condolence \\
\hline $\log$ (length) & $0.29^{* * *}$ & $0.44^{* * *}$ \\
\hline conv. depth & $0.09^{* * *}$ & $0.02^{* * *}$ \\
\hline score of post & $0.0000^{* * *}$ & $0.0000^{*}$ \\
\hline comment age (hour) & $-0.01^{* * *}$ & $-0.01^{* * *}$ \\
\hline Female author & -0.02 & $0.10^{* *}$ \\
\hline Male author & 0.01 & -0.06 \\
\hline distress rating & $-0.08^{* * *}$ & $0.55^{* * *}$ \\
\hline Topic: POSSESSIONS & $0.16^{* * *}$ & -0.09 \\
\hline Topic: POLITICS & $0.69^{* * *}$ & 0.01 \\
\hline Topic: MovING & $0.24^{* * *}$ & -0.02 \\
\hline Topic: DATING & $0.23^{* * *}$ & $0.74^{* * *}$ \\
\hline Topic: VIDEO GAMES & $0.24^{* * *}$ & $-0.50^{* * *}$ \\
\hline Topic: MEDICAL & $0.38^{* * *}$ & $1.54^{* * *}$ \\
\hline Topic: FAMILY & $0.10^{* * *}$ & $1.83^{* * *}$ \\
\hline Topic: SELF REFLECTION & $0.36^{* * *}$ & $0.72^{* * *}$ \\
\hline Topic: VIDEO GAMES 2 & $0.19^{* * *}$ & $-0.46^{* * *}$ \\
\hline Topic: CAR ACCIDENTS & $0.05^{* *}$ & $0.37^{* * *}$ \\
\hline Topic: DEATH & $0.18^{* * *}$ & $0.59^{* * *}$ \\
\hline Topic: FINANCES & $0.36^{* * *}$ & 0.17 \\
\hline Topic: COLLEGE & $0.39^{* * *}$ & $0.23^{* *}$ \\
\hline Topic: SPORTS & $0.23^{* * *}$ & $-0.43^{* * *}$ \\
\hline Topic: DEPRESSION & $0.40^{* * *}$ & $0.90^{* * *}$ \\
\hline Topic: PETS & $0.12^{* * *}$ & $1.59^{* * *}$ \\
\hline Topic: DIET & $0.33^{* * *}$ & -0.20 \\
\hline Topic: ADVICE & $0.26^{* * *}$ & $0.23^{* *}$ \\
\hline Topic: DEATH 2 & $0.34^{* * *}$ & $0.50^{* * *}$ \\
\hline Emotion: fear & $0.33^{* * *}$ & $1.53^{* * *}$ \\
\hline Emotion: anger & $0.23^{* * *}$ & -0.55 \\
\hline Emotion: trust & -0.08 & $-0.81^{* * *}$ \\
\hline Emotion: surprise & $0.16^{* *}$ & $-1.12^{* * *}$ \\
\hline Emotion: positive & $-0.24^{* * *}$ & -0.48 \\
\hline Emotion: negative & -0.01 & 0.07 \\
\hline Emotion: sadness & $0.22^{* * *}$ & $2.56^{* * *}$ \\
\hline Emotion: disgust & $-0.27^{* * *}$ & -0.25 \\
\hline Emotion: joy & $-0.35^{* * *}$ & $-1.37^{* * *}$ \\
\hline 1st person pronouns & $-0.01^{* * *}$ & $0.01^{* * *}$ \\
\hline 2nd person pronouns & $0.04^{* * *}$ & $-0.02^{* * *}$ \\
\hline 3rd person pronouns & $-0.01^{* * *}$ & $-0.004^{*}$ \\
\hline intercept & $-1.08^{* * *}$ & $-7.93^{* * *}$ \\
\hline
\end{tabular}

Table 2: When expressing distress, the effect of social, contextual, and linguistic factors on receiving any reply to distress (left) versus receiving condolence (right).

as not all replies are actually supportive.

\section{The Structure of Condolence}

Individuals regularly employ a common set of strategies in condolence (e.g., Davidowitz and Myrick, 1984; Lehman et al., 1986; Burleson, 2003), from trope-like expressions ("sorry for your loss") to thoughtful and empathetic statements that validate the other's experience. These statements often fall along a spectrum of person-centeredness (High and Dillard, 2012) with respect to their acknowledgment, understanding, and legitimization of the distressed person's state. Here, we analyze the structure of Reddit condolences to examine reg- 
ularities in strategies individuals employ in crafting their responses. We use a data-driven approach to identify themes by fitting a 20-topic LDA model to identify broad themes; to test for structure, we measure the probability of each topic in the sequence of sentences for condolences of different lengths.

Results Condolences follow regular patterns in their strategies for support. Figure 3 shows the presence of different topics by position in the sentence across condolences of different lengths; the most probable words for each topic are listed in Table 3. Three notable trends occur, showing increasing focus on the person experiencing distress.

First, sympathy features prominently in shorter condolences, which focus largely on acknowledging the person's suffering as a result of the distress. These comments serve as bookends to the overall statement, but largely disappear in longer condolences. The use of swearing in these contexts acts not only as an intensifier in expressing the speaker's perception of unpleasantness but also as a way of expressing solidarity through emphasizing in-group membership by transgressing social norms (Fägersten, 2012; Stapleton, 2010).

Second, as condolences become longer, individuals begin adding their own experience within the response (PERSONAL EXPERIENCE). This behavior features prominently in middle-length condolences that still begin with sympathy and then try to relate their own personal experience to that of the suffering. At a high-level, these experiences aim to help the person experiencing distress reframe their own mindset and correspond to a higher-level of person-centeredness (Servaty-Seib and Burleson, 2007; High and Dillard, 2012).

Finally, the longest condolences contain significant amounts of advice and reframing, with less focus on the condolence giver. These condolences can correspond to even higher levels of personcenteredness by trying to engage with the other's experience through advice.

\section{Empathy in Condolence}

At a high level, empathy requires a person to imagine the experience of another as they felt it-to put themselves in the other's shoes. In condolence, empathy provides a powerful, person-centered framing for validating and connecting with those in distress. Distressed individuals have found empathetic condolences more supportive than sympathetic messages (Davidowitz and Myrick, 1984;

\begin{tabular}{ll}
\hline ESTATE & you your they can money them estate their pay funeral \\
SADNESS & rip rest peace crying i'm you're onions you man missed \\
TRAVEL & you they your can car them when back their fire \\
SPORTS & his game him team fan fans they when play hit \\
DIETING & you your can yourself care time good when day don't \\
MUSIC & his made time when song love story it's cry music \\
VIDEO GAMES & game you play games your can they playing when time \\
BODY & his him back when they eyes head face their you \\
PERSONAL EXP. & his him when years time dad died family day ago \\
PETS & your you dog loss him they lost love cat life \\
SHOOTINGS & people they their our tragedy them gun thoughts country \\
MEDICAL & you they can your help health pain doctor mental care \\
RELATIONSHIPS & you your him they can his them yourself their dodged \\
SYMPATHY & you your loss i'm hope hear love man family god \\
CURSING & man i'm you shit made sad fuck fucking cry damn \\
MEMES & amp you respects pay press sad post play alexa your \\
RELIGION & you god our they your their his people life can \\
ADVICE & you your can yourself time feel life help don better \\
SCHOOL & you your work school job can time they good college \\
ADVICE2 & you it's don't your i'm you're people can they them \\
\hline
\end{tabular}

Table 3: Topics for condolence speech reveal broad themes around types of distress (e.g., MEDICAL) as well as condolence strategies (e.g., SYMPATHY)

Shapiro, 2001) and more effective in clinical settings at helping the distressed resolve their emotions (Worden et al., 2018).

Empathy itself has many varying definitions in social psychology (Basch, 1983; Cuff et al., 2016) and the limited computational work employing empathy has largely focused only on mirroring emotional state as a way of empathizing (Collins, 2014; Litvak et al., 2016; Fung et al., 2016; Khanpour et al., 2017). More recently Abdul-Mageed et al. (2017) and Buechel et al. (2018) have gone beyond these simple models to develop and use a corpus for distress and empathy in reactions to news stories. These works adopt a broader definition drawn from multiple sources of empathy which mixes empathy with related concepts of compassion, altruism, and prosocial behavior. (Batson et al., 1987; Sober and Wilson, 1999; Goetz et al., 2010; Mikulincer and Shaver, 2010). In this work, we adopt a stricter definition of measuring empathy based on appraisal theory (Lamm et al., 2007; Wondra and Ellsworth, 2015). Here, empathy occurs when an observer appraises a person's situation in the same way as the person experiencing the distress. This definition more closely mirrors the person-centeredness of the response in terms of how the observer acknowledges and validates different aspects of the distressed person's mental state. Following this definition, we create a new corpus around appraisalbased empathy and develop a classifier that can be used to label condolences for their empathy.

Data and Annotation Distress-condolence pairs were sampled from the Reddit dataset. Condolence 


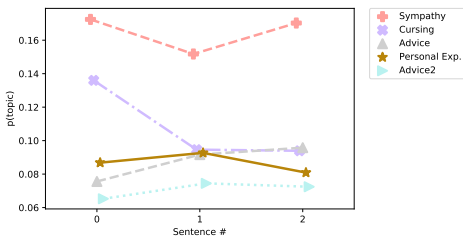

(a) 3 Sentences

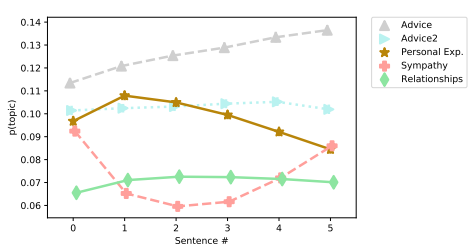

(d) 6 Sentences

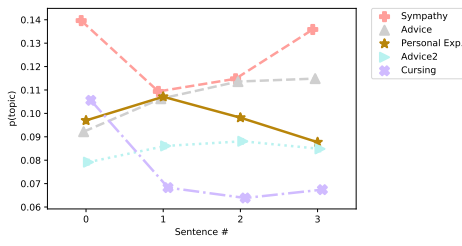

(b) 4 Sentences

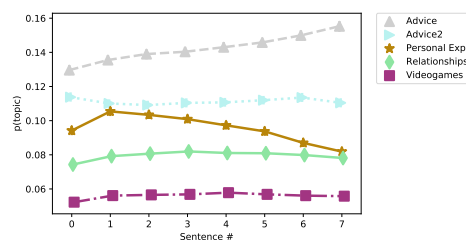

(e) 8 Sentences

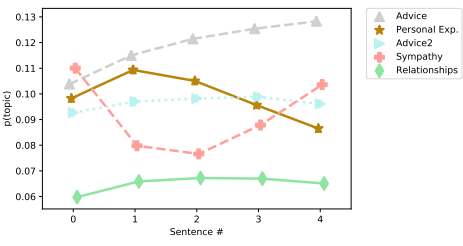

(c) 5 Sentences

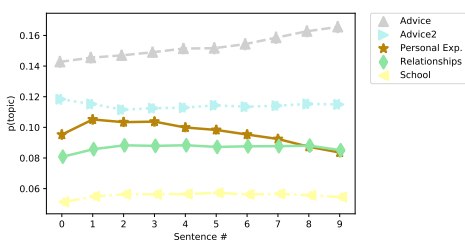

(f) 10 Sentences

Figure 3: Plots of sentence-level topic distribution across condolences of different lengths reveal categorically different strategies (Topics described in Table 3). Shorter condolences focus on expressing sympathy, middlelength include more personal experience, and longer condolences offer substantial amounts of advice.

lengths followed a log-log-normal distribution, and shorter condolences tended to be trite or repetitive, e.g., "so sorry to hear." To introduce diversity in the annotated condolence data, we binned comment pairs by condolence length using Jenks optimization, then reweighted the probability of sampling from each bin to flatten the distribution of lengths. Two annotators identified a set of 1000 distress comments with a self-contained message, without being shown the condolence to avoid bias. Individuals may express their distress over multiple comments in a discussion thread, so this process was aimed at reducing the prior context needed to estimate appraisal to a single distress comment.

Annotators were shown a condolence reply to a comment and asked to rate on a five-point Likert scale to what degree did the observer appraise the other person's situation in the same way along the following dimensions: (1) pleasantness, (2) anticipated effort in dealing with the situation, (3) situational control, (4) how much oneself or another person was responsible for the situation, (5) attentional activity, and (6) certainty about what was happening in the situation or what would happen next. High scoring comments acknowledge and validate the distressed person's experience.

Prior to annotating the full dataset, annotators collaboratively developed guidelines and completed five rounds of training on 100 items of heldout data in each round and discussed each case of disagreement. Annotators attained Krippendorff's $\alpha \approx 0.6$ for the final two rounds. Following training and adjudication, the final 1,000 condolence replies were annotated. After an initial pass, Krippendorff's $\alpha$ was 0.359 . While this initial value seems low, $\alpha$ is strongly affected by the large class skew from most condolences not being empathetic (score 1). A second pass was made across the 25 comment pairs where annotators disagreed by 3 or more points, where annotators discussed their disagreements and updated their individual ratings, after which $\alpha=0.431$; these disagreements were largely due to unintentional mistakes or misinterpretations, rather than substantive disagreements on empathy. In the final dataset, annotators differentiated by at most one scale point on $91.2 \%$ of the items (Pearson $r=0.58$ ). While the agreement value is moderate, it matches similar agreement levels seen when annotation requires inferring mental states and intentions from text (e.g., Card et al., 2015; Rashkin et al., 2016; Rashid and Blanco, 2017; Breitfeller et al., 2019). The difficulty of annotation stems from interpreting the intentions, appraisals, and alignment between the distress comment and observer's comment. Further, the choice to diversity the data by sampling across longer replies likely depressed agreement, as shorter replies often are low-empathy (e.g., trite messages) which annotators readily agreed on. The final empathy rating is the mean of the two annotations.

Recognizing Empathy Two types of regression models were trained for predicting the empathy rating of a condolence using our dataset, which use either the target's and observer's texts or just 
the observer's text. The first type of models uses a random forest regressor that is trained on unigram and bigrams of the target and observers comments, using separate feature spaces for each. The second type of model uses RoBERTa (Liu et al., 2019) as a base, starting from the pretrained roberta-base parameters. When using the target and observer text as inputs, the texts are separated by the [SEP] token. The [CLS] representations of each input were concatenated and passed through a fully-connected linear layer, using sigmoid activation to bound the output value in $[1,5]$. Due to the empathy rating imbalance in the data, we construct randomized stratified partitions for training $(80 \%)$, validation $(10 \%)$, and test $(10 \%)$ using the rounded value of the empathy rating. Models are compared with the mean empathy rating.

Both models surpassed the baseline of predicting the mean value from the training data, as seen in Table 4 , with the RoBERTa models performing best. ${ }^{2}$ For both the RoBERTa and Random Forest models, knowledge of the target's comments improved performance, suggesting that models benefit from being able to align the two inputs in determining empathy. Nonetheless, performance of the best model is moderate at best and we view these results as a preliminary step at identifying appraisal-based empathy in text.

As a follow-up analysis, we used the Target $\&$ Observer model to rate unlabeled condolence replies and manually examined a random 100 responses rated with empathy $\geq 2$, which signals more than the minimal empathetic alignment. Of these replies, $84 \%$ contained at least two empathetic alignments (e.g., aligning with the target's perception of pleasantness and situational control), suggesting the model is effective at recognizing empathetic speech and any misclassifications are more likely to be underestimates of empathy.

As a further comparison, we computed the empathy scores for the model of Buechel et al. (2018) on our data; the two scores had a Pearson $r=0.343$, indicating that, while related, both are capturing substantially different notions of empathy.

\footnotetext{
${ }^{2}$ Additional RoBERTa models were trained using a language model that had first been fine-tuned using masked language modeling on the distress and condolence comments for 10 epochs; however, these models resulted in slightly worse performance: the Observer-only had MSE $=0.561$ and $R^{2}=0.082$ and the Target \& Observer model had MSE $=0.516$ and $R^{2}=0.156$
}

\begin{tabular}{lcc} 
& MSE & \multicolumn{1}{c}{$R^{2}$} \\
\hline Baseline: mean value & 0.565 & -0.008 \\
Random Forest: Target \& Obs. & 0.492 & 0.128 \\
Random Forest: Obs. Text Only & 0.517 & 0.044 \\
RoBERTa: Target \& Obs. & $\mathbf{0 . 4 2 9}$ & $\mathbf{0 . 2 9 7}$ \\
RoBERTa: Obs. Text Only & 0.555 & 0.094
\end{tabular}

Table 4: Empathy model performances

\section{What Makes a Good Condolence?}

Not all condolences are equally effective at offering support. Multiple works on bereavement have surveyed the effectiveness of different condolences (Burleson, 2009), noting that many fall along a spectrum of helpfulness to the distressed. For example, individuals typically find empathetic and validating comments more helpful, unlike advice or trope-like messages (Davidowitz and Myrick, 1984; Lehman et al., 1986; Rack et al., 2008). Here, we build a logistic regression model to evaluate which condolences Redditors found helpful and identify what features make for effective condolences.

\subsection{Data}

Authors of distress comments occasionally respond to condolence comments, which can include acknowledgment of the helpfulness of the condolence, e.g., "your comment made my day." We identify all such responses and treat the 23,301 paired condolences as positive examples of a good condolence. As negative examples, we use all remaining 149,992 condolence comments that did not receive such a reply. While some of the negative examples are likely effective condolences, these false negatives only result in an underestimate of the effect of the explanatory coefficients.

\subsection{Model and Features}

Condolence effectiveness is modeled using a nested-effects logistic regression with the dependent variable of whether the condolence was responded to with gratitude. Random effects are added for the subreddit with a nested effect for condolences made to posts receiving 30 or more replies; posts receiving fewer are modeled with a common nested effect. Note that these random effects control for relative differences in the level of gratitude and behavioral norms in each subreddit, allowing more accurate estimates of which content features contribute to effective condolences. Three groups of regression features were selected: 
two from theory for known helpful and unhelpful strategies, with an additional group of data-driven controls, all described next.

Helpful Strategy Features In the first group, we include the macro-empathy estimates of Buechel et al. (2018) and our appraisal-based empathy estimate of the comment, as person-centered empathetic responses are known to be more helpful in clinical therapy (Nienhuis et al., 2018). As a third test, we include uses of first-, second-, and third-person pronominal referents from LIWC (Pennebaker et al., 2001). Increased use of each pronoun category reflects narrative focus on the condoler, distressed person, or the situation being described, respectively; in particular, mentions of the distressed person are more aligned with a person-centered message. Fourth, individuals will mirror the language as a way of decreasing social distance which can increase trust (Scissors et al., 2008); Wang et al. (2015) found that lexical alignment is associated with increased emotional support. Therefore, we include a feature for lexical alignment as the \% of the condolence's words that were also used in the distress comment.

Unhelpful Strategy Features Some wellintentioned responses may include strategies that are unhelpful in practice. Lehman et al. (1986) note that forced positivity in the face of distress is often viewed poorly; therefore, to test this effect, we include a sentiment estimate of the condolence using VADER (Hutto and Gilbert, 2014). Similarly, minimizing phrases such as "it's not that bad" or "I'm sorry you feel sad" invalidate the experience and emotions of the distressed persons (Lehman et al., 1986; Hogan et al., 1994); to test for these effects, we include the presence of a list of such phrases drawn from observational studies and matched using regular expressions. Third, we include a separate minimizing phrase for trivializing "just” (Kiesling, 2011)—e.g., "it's just an exam"-which is modeled by identifying the presence of an adverbial use in the text.

Control Features As controls, we include (i) the topics of the condolence (Table 3), which act as coarse proxies of the strategy and content, (ii) the score of the comment containing the distress and the time between the distress comment and condolence reply (minutes), (iii) the length of the condolence, and (iv) temporal factors for the month, day of week, and hour of day. Finally, multiple stud-

\begin{tabular}{lc}
\hline Topic: PERSONAL EXPERIENCE & $-0.55^{* *}$ \\
Topic: SYMPATHY & $-0.91^{* * *}$ \\
Topic: CURSING & $-0.77^{* * *}$ \\
Topic: RELIGION & $-1.00^{* * *}$ \\
Topic: ADVICE & $-1.21^{* * *}$ \\
Topic: ADVICE2 & 0.22 \\
Post score & $0.0000^{* * *}$ \\
Reply delay (min) & $-0.001^{* * *}$ \\
log(condolence length) & $0.15^{* * *}$ \\
Female author & 0.04 \\
Male author & $-0.12^{* * *}$ \\
Sentiment & $0.25^{* * *}$ \\
Has adverbial “just"? & -0.01 \\
Has minimization? & $-0.15^{* * *}$ \\
Buechel et al. (2018) empathy & $0.18^{* * *}$ \\
Appraisal-based empathy & $-0.17^{* * *}$ \\
Lexical alignment & $0.37^{* * *}$ \\
\# First person pronouns & 0.001 \\
\# Second person pronouns & $0.05^{* * *}$ \\
\# Third person pronouns & $-0.05^{* * *}$ \\
Constant & $-2.72^{* * *}$ \\
\hline Note: ${ }^{*} p<0.1$; ${ }^{* *} p<0.05 ;{ }^{* * *} p<0.01$ \\
\end{tabular}

Table 5: Coefficients for predicting whether a condolence will receive gratitude; for simplicity, coefficients for temporal controls and topics corresponding to experiential themes (e.g., sports) are omitted and provided in supplemental section D.

ies have reported gender differences in strategies of support, with women typically offering more emotionally complex and empathetic condolences (Knight et al., 1998; Rack et al., 2008; Burleson et al., 2009); to test for this effect, we include the gender prediction from genderperformr.

\subsection{Results}

The linguistic factors associated with helpful condolences largely followed expectations from observational studies, with one significant exception. As predicted from observational studies, condolences with markers of person-centered responses were rated as more helpful, which included lexical alignment and narrative focus on the other person (second-person pronouns). In annotation, we observed that condolences shift between the "personal you" of the distress person and use of the "generic you," which is known to be evoked in meaning making (Orvell et al., 2017, 2019); given the positive coefficient for second-person pronouns, future work may attempt to distinguish between these uses to test whether such meaning-making comments contribute to more effective condolence.

Also predicted, advice is strongly negative 
to good condolence-despite being the most commonly-used strategy (cf. Figure 3). Replies with the ADVICE 2 topic contained more references to third parties than ADVICE; some of these included popular supportive quotes, not actually condolence, or assessment of and advice for a thirdparty outside of the interaction being modeled in this regression. Similarly, sympathy and invocations of religious language (which we found often contains minimizing tropes) are known to be found less helpful and have negative coefficients here as well. Last, our study confirms the expected disparity for men and women in condolence helpfulness.

However, our results disagree with prior observations on empathy and we find that, while the compassion-like empathy of Buechel et al. (2018) is found helpful, condolences with the more personcentered appraisal-based empathy were less likely to receive gratitude. We speculate that people may turn to Reddit for lighter, less-personal forms of support in times of distress, whereas the more compassion-like empathy of Buechel et al. (2018) is helpful when more personal responses are not licensed by the relative anonymity of the platform. ${ }^{3}$

Our results also disagree with expectations around forced positivity (Lehman et al., 1986), where positive sentiment replies are consistently more helpful. We interpret this result pointing to a different goal of support by Reddit users who seek out positive reinforcement, rather than comments that require emotional effort to engage with complex emotions.

While we are only able to speculate on negative impact of appraisal-based empathy, the effect could be due to different goals for the desired support received online, where individuals seek out information instead of empathy (Yao et al., 2015). Alternatively, here, we have modeled condolence helpfulness using a fixed set of phrases to identify thanks in replies; it could be that the more empathetic responses generate replies that, while not containing these thanks-expressions, still signal the condolence's positive utility. Our results motivate future work to understand online users' preferences for empathy in support: as millions of people already respond to distress with good intentions each year, improving these supportive efforts has the

\footnotetext{
${ }^{3}$ As a follow-up analysis, we also tested whether a binary encoding of higher appraisal empathy (score $\geq 2$ ) instead of a continuous marker would be found to be more helpful; after re-running the regressions, the appraisal-based empathy still had a negative coefficient.
}

potential to better the lives of millions.

\section{Ethics}

Distress is inherently personal and computational studies on such matters warrant ethical consideration. In weighing the risks and benefits of our studies, the largest risk has been the loss of privacy, as individuals expressing their distress may have contextual expectations of privacy or anonymity (Fiesler and Proferes, 2018). To mitigate this risk, we report only paraphrased examples and aggregate statistics. Further, we only release this data to researchers upon request and provided they follow similar privacy practices. As a counter balance, this study has considerable benefit by providing better information on what makes for effective condolences; the insights from this study can be distilled into practical advice that can make for more supportive online communities.

\section{Conclusion}

Distress is an omnipresent part of life, and individuals turn to their social circle and social platforms for support when experiencing it. In this paper, we have developed new computational models for recognizing distress, condolences to that distress, and empathy within condolence. Applying those models, we examine the dynamics of distress and condolence, showing that not all distress is treated equally online, and there exist regular structures within condolence. Through analyzing millions of condolence responses, we test what makes for effective condolence online, showing that while some features predicted from observation studies hold true online, e.g., increasing person-centeredness of the message (High and Dillard, 2012), distressed individuals did not find empathetic comments more helpful, suggesting different goals from online support. Our results have important implications for (i) individuals by providing concrete suggestions of how to express one's distress to make it more likely to receive support, (ii) site operators by allowing them to observe the emotional health and responsiveness of their community, potentially reaching out to underserved individuals who have yet to receive support, and (iii) the general public for authoring more effective supportive messages. Models and reproducible code are available at https://blablablab.si. umich.edu/projects/condolence/ and data is made available upon request. 


\section{Acknowledgments}

The authors thank members of the Blalablab for their helpful feedback, the anonymous reviewers for their thoughtful insight which definitely made this paper better, and the highly empathetic Redditors for restoring our faith in the goodness of humanity. This material is based upon work supported by the National Science Foundation under Grants No IIS-1850221 and IIS-2007251. The last author was supported in part through the Amazon Alexa Prize Competition 3.

\section{References}

Muhammad M Abdul-Mageed, Anneke Buffone, Hao Peng, Johannes Eichstaedt, and Lyle Ungar. 2017. Recognizing pathogenic empathy in social media. In Eleventh International AAAI Conference on Web and Social Media.

Michael Franz Basch. 1983. Empathic understanding: A review of the concept and some theoretical considerations. Journal of the American Psychoanalytic Association, 31(1):101-126.

C Daniel Batson, Jim Fultz, and Patricia A Schoenrade. 1987. Distress and empathy: Two qualitatively distinct vicarious emotions with different motivational consequences. Journal of personality, 55(1):19-39.

Prakhar Biyani, Cornelia Caragea, Prasenjit Mitra, and John Yen. 2014. Identifying emotional and informational support in online health communities. In Proceedings of COLING 2014, the 25th International Conference on Computational Linguistics: Technical Papers, pages 827-836.

Luke Breitfeller, Emily Ahn, David Jurgens, and Yulia Tsvetkov. 2019. Finding microaggressions in the wild: A case for locating elusive phenomena in social media posts. In Proceedings of the 2019 Conference on Empirical Methods in Natural Language Processing and the 9th International Joint Conference on Natural Language Processing (EMNLPIJCNLP), pages 1664-1674.

Jed R Brubaker and Gillian R Hayes. 2011. " we will never forget you [online]" an empirical investigation of post-mortem myspace comments. In Proceedings of the ACM 2011 conference on Computer supported cooperative work, pages 123-132.

Jed R Brubaker, Funda Kivran-Swaine, Lee Taber, and Gillian R Hayes. 2012. Grief-stricken in a crowd: The language of bereavement and distress in social media. In Sixth International AAAI Conference on Weblogs and Social Media.

Sven Buechel, Anneke Buffone, Barry Slaff, Lyle Ungar, and João Sedoc. 2018. Modeling empathy and distress in reaction to news stories. In Proceedings of EMNLP.
Brant R Burleson. 2003. Emotional support skills. Handbook of communication and social interaction skills, pages 551-594.

Brant R Burleson. 2009. Understanding the outcomes of supportive communication: A dual-process approach. Journal of Social and Personal Relationships, 26(1):21-38.

Brant R Burleson, Lisa K Hanasono, Graham D Bodie, Amanda J Holmstrom, Jessica J Rack, Jennifer Gill Rosier, and Jennifer D McCullough. 2009. Explaining gender differences in responses to supportive messages: Two tests of a dual-process approach. Sex Roles, 61(3-4):265.

C Daryl Cameron, Cendri A Hutcherson, Amanda M Ferguson, Julian A Scheffer, Eliana Hadjiandreou, and Michael Inzlicht. 2019. Empathy is hard work: People choose to avoid empathy because of its cognitive costs. Journal of Experimental Psychology: General.

Dallas Card, Amber E. Boydstun, Justin H. Gross, Philip Resnik, and Noah A. Smith. 2015. The media frames corpus: Annotations of frames across issues. In Proceedings of ACL.

P Cattell, James. 1955. The holiday syndrome. Psychoanalytic Review, 42(1):39-43.

Franklin M Collins. 2014. The relationship between social media and empathy. Master's thesis, Georgia Southern University.

Benjamin MP Cuff, Sarah J Brown, Laura Taylor, and Douglas J Howat. 2016. Empathy: A review of the concept. Emotion Review, 8(2):144-153.

Mitchell Davidowitz and Robert D Myrick. 1984. Responding to the bereaved: An analysis of helping statements. Death Education, 8(1):1-10.

Munmun De Choudhury and Sushovan De. 2014. Mental health discourse on reddit: Self-disclosure, social support, and anonymity. In Eighth international AAAI conference on weblogs and social media.

Munmun De Choudhury and Emre Kiciman. 2017. The language of social support in social media and its effect on suicidal ideation risk. In Proc. ICWSM, pages 32-41.

Jacob Devlin, Ming-Wei Chang, Kenton Lee, and Kristina Toutanova. 2019. Bert: Pre-training of deep bidirectional transformers for language understanding. In Proceedings of NAACL.

Katrin Döveling. 2017. Online emotion regulation in digitally mediated bereavement. why age and kind of loss matter in grieving online. Journal of Broadcasting \& Electronic Media, 61(1):41-57.

Kristy Beers Fägersten. 2012. Whos swearing now? The social aspects of conversational swearing. Cambridge Scholars Publishing. 
Casey Fiesler and Nicholas Proferes. 2018. "participant" perceptions of twitter research ethics. Social Media + Society, 4(1):2056305118763366.

Pascale Fung, Dario Bertero, Yan Wan, Anik Dey, Ricky Ho Yin Chan, Farhad Bin Siddique, Yang Yang, Chien-Sheng Wu, and Ruixi Lin. 2016. Towards empathetic human-robot interactions. In International Conference on Intelligent Text Processing and Computational Linguistics, pages 173-193. Springer.

Xavier Glorot and Yoshua Bengio. 2010. Understanding the difficulty of training deep feedforward neural networks. In Proceedings of the Thirteenth International Conference on Artificial Intelligence and Statistics, volume 9 of Proceedings of Machine Learning Research, pages 249-256, Chia Laguna Resort, Sardinia, Italy. PMLR.

Jennifer L Goetz, Dacher Keltner, and Emiliana SimonThomas. 2010. Compassion: an evolutionary analysis and empirical review. Psychological bulletin, 136(3):351.

Morton G Harmatz, Arnold D Well, Christopher E Overtree, Kathleen Y Kawamura, Milagros Rosal, and Ira S Ockene. 2000. Seasonal variation of depression and other moods: a longitudinal approach. Journal of biological rhythms, 15(4):344-350.

Andrew C High and James Price Dillard. 2012. A review and meta-analysis of person-centered messages and social support outcomes. Communication Studies, 63(1):99-118.

Nancy S Hogan, Lydia De Santis, Alice S Demi, Kathleen V Cowles, and H Miriam Ross. 1994. Things that help and hinder adolescent sibling bereavement. Western Journal of Nursing Research, 16(2):132153

Jina Huh, Leslie S Liu, Tina Neogi, Kori Inkpen, and Wanda Pratt. 2014. Health vlogs as social support for chronic illness management. ACM Transactions on Computer-Human Interaction (TOCHI), 21(4):131.

Clayton J Hutto and Eric Gilbert. 2014. Vader: A parsimonious rule-based model for sentiment analysis of social media text. In Eighth international AAAI conference on weblogs and social media.

Hamed Khanpour, Cornelia Caragea, and Prakhar Biyani. 2017. Identifying empathetic messages in online health communities. In Proceedings of the Eighth International Joint Conference on Natural Language Processing (Volume 2: Short Papers), pages 246-251.

Scott F Kiesling. 2011. Stance in context: Affect, alignment and investment in the analysis of stancetaking. In iMean conference, volume 15.
Kim H Knight, Morton H Elfenbein, and Julie A Messina-Soares. 1998. College students' perceptions of helpful responses to bereaved persons: Effects of sex of bereaved persons and cause of death. Psychological reports, 83(2):627-636.

Karolina Krysinska and Karl Andriessen. 2013. Suicide bereavement online: Sharing memories, seeking support, and exchanging hope. In Suicide prevention and new technologies, pages 150-165. Springer.

Claus Lamm, C Daniel Batson, and Jean Decety. 2007. The neural substrate of human empathy: effects of perspective-taking and cognitive appraisal. Journal of cognitive neuroscience, 19(1):42-58.

Darrin R Lehman, John H Ellard, and Camille B Wortman. 1986. Social support for the bereaved: Recipients' and providers' perspectives on what is helpful. Journal of consulting and clinical psychology, 54(4):438.

Daniel Lim and David DeSteno. 2016. Suffering and compassion: The links among adverse life experiences, empathy, compassion, and prosocial behavior. Emotion, 16(2):175.

Marina Litvak, Jahna Otterbacher, Chee Siang Ang, and David Atkins. 2016. Social and linguistic behavior and its correlation to trait empathy. In Proceedings of the Workshop on Computational Modeling of Peoples Opinions, Personality, and Emotions in Social Media (PEOPLES), pages 128-137.

Yinhan Liu, Myle Ott, Naman Goyal, Jingfei Du, Mandar Joshi, Danqi Chen, Omer Levy, Mike Lewis, Luke Zettlemoyer, and Veselin Stoyanov. 2019. Roberta: A robustly optimized bert pretraining approach. arXiv preprint arXiv:1907.11692.

Lydia Manikonda, Heather Pon-Barry, Subbarao Kambhampati, Eric Hekler, and David W McDonald. 2014. Discourse analysis of user forums in an online weight loss application. In Proceedings of the joint workshop on social dynamics and personal attributes in social media, pages 28-32.

Mario Ed Mikulincer and Phillip R Shaver. 2010. Prosocial motives, emotions, and behavior: The better angels of our nature. American Psychological Association.

Saif M Mohammad and Peter D Turney. 2013. Crowdsourcing a word-emotion association lexicon. Computational Intelligence, 29(3):436-465.

Amit Navindgi, Caroline Brun, Cécile Boulard Masson, and Scott Nowson. 2016. Steps toward automatic understanding of the function of affective language in support groups. In Proceedings of The Fourth International Workshop on Natural Language Processing for Social Media, pages 26-33. 
Jacob B Nienhuis, Jesse Owen, Jeffrey C Valentine, Stephanie Winkeljohn Black, Tyler C Halford, Stephanie E Parazak, Stephanie Budge, and Mark Hilsenroth. 2018. Therapeutic alliance, empathy, and genuineness in individual adult psychotherapy: A meta-analytic review. Psychotherapy Research, 28(4):593-605.

Ariana Orvell, Ethan Kross, and Susan A Gelman. 2017. How "you" makes meaning. Science, 355(6331):1299-1302.

Ariana Orvell, Ethan Kross, and Susan A Gelman. 2019. "you" and "i" in a foreign land: The persuasive force of generic-you. Journal of Experimental Social Psychology, 85:103869.

Fabian Pedregosa, Gaël Varoquaux, Alexandre Gramfort, Vincent Michel, Bertrand Thirion, Olivier Grisel, Mathieu Blondel, Peter Prettenhofer, Ron Weiss, Vincent Dubourg, et al. 2011. Scikit-learn: Machine learning in python. the Journal of machine Learning research, 12:2825-2830.

James W Pennebaker, Martha E Francis, and Roger J Booth. 2001. Linguistic inquiry and word count: LIWC 2001. Mahway: Lawrence Erlbaum Associates, 71(2001):2001.

Jessica J Rack, Brant R Burleson, Graham D Bodie, Amanda J Holmstrom, and Heather Servaty-Seib. 2008. Bereaved adults' evaluations of grief management messages: Effects of message person centeredness, recipient individual differences, and contextual factors. Death Studies, 32(5):399-427.

Farzana Rashid and Eduardo Blanco. 2017. Dimensions of interpersonal relationships: Corpus and experiments. In Proceedings of the 2017 Conference on Empirical Methods in Natural Language Processing, pages 2307-2316, Copenhagen, Denmark. Association for Computational Linguistics.

Hannah Rashkin, Sameer Singh, and Yejin Choi. 2016. Connotation Frames: A Data-Driven Investigation. In Proceedings of the 54th Annual Meeting of the Association for Computational Linguistics (Volume 1: Long Papers), 5, pages 311-321, Stroudsburg, PA, USA. Association for Computational Linguistics.

Norman E Rosenthal, David A Sack, J Christian Gillin, Alfred J Lewy, Frederick K Goodwin, Yolande Davenport, Peter S Mueller, David A Newsome, and Thomas A Wehr. 1984. Seasonal affective disorder: a description of the syndrome and preliminary findings with light therapy. Archives of general psychiatry, 41(1):72-80.

Lauren E Scissors, Alastair J Gill, and Darren Gergle. 2008. Linguistic mimicry and trust in text-based CMC. In Proceedings of ACM conference on Computer Supported Cooperative Work, pages 277-280.

Heather L Servaty-Seib and Brant R Burleson. 2007. Bereaved adolescents' evaluations of the helpfulness of support-intended statements: Associations with person centeredness and demographic, personality, and contextual factors. Journal of Social and Personal Relationships, 24(2):207-223.

Ester R Shapiro. 2001. Grief in interpersonal perspective: Theories and their implications.

Elliott Sober and David Sloan Wilson. 1999. Unto others: The evolution and psychology of unselfish behavior. 218. Harvard University Press.

Karyn Stapleton. 2010. Swearing. Interpersonal pragmatics, 6:289.

Krista K Trobst, Rebecca L Collins, and Jayne M Embree. 1994. The role of emotion in social support provision: Gender, empathy and expressions of distress. Journal of Social and Personal Relationships, 11(1):45-62.

Sida Wang and Christopher D Manning. 2012. Baselines and bigrams: Simple, good sentiment and topic classification. In Proceedings of the 50th annual meeting of the association for computational linguistics: Short papers-volume 2, pages 90-94. Association for Computational Linguistics.

Yafei Wang, John Yen, and David Reitter. 2015. Pragmatic alignment on social support type in health forum conversations. In Proceedings of the 6th Workshop on Cognitive Modeling and Computational Linguistics, pages 9-18.

Zijian Wang and David Jurgens. 2018. Its going to be okay: Measuring Access to Support in Online Communities. In Proc. EMNLP.

Thomas Wolf, Lysandre Debut, Victor Sanh, Julien Chaumond, Clement Delangue, Anthony Moi, Pierric Cistac, Tim Rault, Rmi Louf, Morgan Funtowicz, Joe Davison, Sam Shleifer, Patrick von Platen, Clara Ma, Yacine Jernite, Julien Plu, Canwen Xu, Teven Le Scao, Sylvain Gugger, Mariama Drame, Quentin Lhoest, and Alexander M. Rush. 2019. Huggingface's transformers: State-of-the-art natural language processing. ArXiv, abs/1910.03771.

Joshua D Wondra and Phoebe C Ellsworth. 2015. An appraisal theory of empathy and other vicarious emotional experiences. Psychological review, 122(3):411.

J William Worden et al. 2018. Grief counseling and grief therapy: A handbook for the mental health practitioner. Springer Publishing Company.

Tang Yao, Qiuying Zheng, and Xiucheng Fan. 2015. The impact of online social support on patients quality of life and the moderating role of social exclusion. Journal of Service Research, 18(3):369-383. 


\section{A Condolence and Distress Models}

\section{A.1 Dataset}

Both the condolence and distress datasets are collected using the heuristic method detailed in the paper. An initial set of stereotypical "seed" condolence phrases is augmented by performing the process of retrieving sibling comments to comments containing these condolence phrases and then performing an n-grams analysis to discover other common phrases. This final list of 21 phrases, shown in Table 6 was used to identify distress comments as described in the main paper.

The final condolence dataset had 431,283 positive examples and 430,311 negative examples. The final distress dataset contained 112,265 positive examples and 116,939 negative examples.

When training, the raw text was extracted from markdown, code blocks were removed, links were stripped, and only ASCII characters were kept. Newlines were replaced with a single space.

\section{A.2 BERT Models}

Both deep learning classifiers were fine-tuned on a pretrained BERT model with 12 heads and $110 \mathrm{M}$ parameters, trained on lower case English text (the HuggingFace bert-base-uncased model), and share the same architecture and training method.

For both models, we feed 768-long hidden output into a fully-connected layer with 2 outputs, which are then fed through a softmax activation function.

During training, a dropout with probability 0.5 was added between the BERT output and the fully connected layer. The fully-connected layer was initalized using Xavier initialization (Glorot and Bengio, 2010). ADAM optimizer was used to minimize cross-entropy loss with learning rate 0.001 for the fully connected layer and 0.00001 for BERT parameters initially, and decreased by a factor of 10 every three epochs. The training set was shuffled every epoch. The models were trained overnight with batches of 16 comments on a single NVIDIA GTX $1080 \mathrm{Ti}$.

\section{A.3 SVM Classifiers}

SVM classifiers are trained as a baseline for comparison. The inputs are preprocessed in the same way (text extracted from markdown, links and code blocks stripped, and Unicode symbols removed). Again, both the condolence and distress classifiers

\begin{tabular}{ll}
\hline "made me tear up" & "you dodged a bullet" \\
"take care of yourself" & "even begin to imagine" \\
"my heart goes out" & "not beat yourself up" \\
"please take care of" & "keep your head up" \\
"heart goes out to" & "can not even begin" \\
"do not blame yourself" & "hope you find peace" \\
"my thoughts and prayers" & "there are no words" \\
"this made me cry" & "remember the good times" \\
"my deepest condolences" & "can not imagine losing" \\
"can not even imagine" & "god bless you and" \\
"sorry for your loss" & \\
\hline
\end{tabular}

Table 6: The stereotypical condolence phrases used to identify distress comments in the initial dataset collection process.

\begin{tabular}{rll} 
& Test & Validation \\
\hline Distress SVM & 0.629 & 0.617 \\
Condolence SVM & 0.829 & 0.830 \\
Distress BERT & 0.714 & 0.717 \\
Condolence BERT & 0.844 & 0.846
\end{tabular}

Table 7: Table of model accuracies on train and test splits

were trained the same way with the same hyperparameters.

The same random seed is set as when training the deep learning models, so the training, validation, and test datasets are the same between the BERT and SVM classifiers. We trained the linear SVM on comments count-encoded with the 50,000 most common uni- and bigrams. Each classifier took a few minutes to train.

Table 7 shows test and validation accuracies for all four models, and Table 8 shows test and validation F-1 scores for all four models.

\section{B Empathy Model}

\section{B.1 Dataset}

The dataset was collected as detailed in the paper, then cleaned to be stripped of markdown, links, and images.

\section{B.2 Random Forest Regressor}

A random forest regressor is trained to predict empathy (as an average of the two annotator scores) given unigram and bigram features of either (i) only the Observer's condolence reply as input or (ii) the Target's comment and Observer's reply. When both the Observer's and Target's texts are used, separate features are used to record the presence of unigrams and bigrams in each. The random forest has 100 estimators using the default parameters from Scikit 


\begin{tabular}{rll} 
& Test & Validation \\
\hline Distress SVM & 0.617 & 0.604 \\
Condolence SVM & 0.815 & 0.815 \\
Distress BERT & 0.714 & 0.707 \\
Condolence BERT & 0.844 & 0.833
\end{tabular}

Table 8: Table of model F-1 Scores on train and test splits

Learn 0.21.3 (Pedregosa et al., 2011). Training the regressor on $80 \%$ of the annotated dataset took approximately 10 minutes.

\section{B.3 Deep Learning Model}

Two RoBERTa (Liu et al., 2019) models were trained on the same dataset as the random forest model using the roberta-base set of parameters to initialize. Models were trained either providing (i) only the Observer's condolence reply as input or (ii) the Target's comment and Observer's reply. In the latter case, the two texts are separated by the [SEP] token. In both cases, classification is done using the [CLS] token. Both RoBERTa models were implemented using the simpletransformers package using the default hyperparameters, including learning rate $4 \mathrm{e}$ 5, batch size 8, and Adam $\epsilon=1 \mathrm{e}-8$. Models were trained for 20 epochs Each model is trained on a single NVIDIA GTX 1080 Ti graphics card, and took about 30 minutes for 20 epochs. No hyperparameter tuning was performed and performance is reported over a single run using a fixed seed.

\section{Topic Modeling}

For both distress and condolence comments, we trained LDA topic models using MALLET using its default hyperparameters for all options and using 20 topics to reflect high-level themes in the data. To preprocess, we stripped markdown, images, and links. We show the top 20 words associated with each topic, as well the topic label we decided, in Table 9 for distress topics and Table 10 for condolence topics.

\section{Regression Experiments}

We run mixed effects regressions for several experiments: predicting whether a distress comment receives any response, predicting whether a distress comment receives a condolence response, and predicting whether a condolence comment receives an appreciative response from the distressed individ- ual. In measuring helpful condolences, the expressions described in Table 11 were used to recognize minimizing condolences.

In these regressions, temporal controls were included, but were excluded in the regression output in the main paper. We include all regression results, including the controls, here. Table 12 shows regression results for receiving any reply (left) and the results for receiving a condolence reply (right). Finally, Table 13 shows regression results for what condolence receives a reply expressing gratitude (i.e., a helpful condolence). 


possessions
politics
moving
dating
video games
medical
family
nighttime
self reflection
dieath2
video games 2
car accidents
diances
college

car they bought them years when sold back it's buy lost ago can mine put you good i've time year they people you their them don't i'm it's our can family his when country your fucking shit trump world children

live years they you miss back city our lived moved home year area ago family town people place living house

him his when told time friend friends didn't guy girl years back wanted asked day our months started school talk

game play i'm games phone i've when it's tried playing time amp can back they bought played can't lost computer

they doctor cancer hospital years pain surgery weeks can months when you days back time heart week ago i'm day

me his him when dad years mom they family kids died them mother parents our brother time year father sister wife

when back his day him home night they house time room told our didn't work door left them bed asked

i'm it's i've don't time i'll feel can't work myself good yeah that's trying can day gonna you hard life

game killed play playing time died they lost when them team played i'm i've him times back can level good

his car him hit when back killed died guy head shot they accident dead didn't fell friend left driving time

his died him miss when dead death show they time years man killed love god he's passed great himself favorite

they them sold money back bought lost account when buy ago card their week today sell didn't days time can

job work i'm money school year years time pay can college working they life make back our don't good afford

him team game year his fan i'm season our miss he's lost play good week they win games fucking back

feel you life can don myself time things people when better lot help depression love years they him make them

dog him cat they when them his our dogs cats years vet died home put day time miss back ago

eat food day eating i'm weight week can water lost i've when good them you ate time made lbs make

you your i'm can it's don't appreciate time help people advice good feel lot you're i've make hope trying i'll

you fucking i'm shit dead fuck man lol gonna yeah miss day god died post damn life can edit die

Table 9: Labels and most probable words for distress topics 


estate / legal
sad emotions
traveling
sports
dieting
movies / song
video games
body parts
personal exp.
pets
advice
shootings
meligion
medical

you your they can money them estate their pay funeral insurance family make death account his don't help lawyer when

rip rest peace crying i'm you're onions you man missed hug godspeed sweet cutting sad his damn brother prince easy

you they your can car them when back their fire time hear happened it's people phone bike work drive area

his game him team fan fans they when play hit our head year players player season good time great win

you your can yourself care time good when day don't eat make back it's body head food weight work try

his made time when song love story it's cry music scene show great movie sad emotional episode tear game feels

game you play games your can they playing when time players them people good team played don't back it's player

his him back when they eyes head face their you man time them our hand black light home looked left

his him when years time dad died family day ago lost year they passed friend back mom life friends didn't

your you dog loss him they lost love cat life when our i'm years them time loved dogs good heart

people they their our tragedy them gun thoughts country prayers can trump when it's shooting don't guns his mass school

you they can your help health pain doctor mental care when time i'm weeks hospital baby years medical months people

you your him they can his them yourself their dodged bullet care people relationship when don't person make life child

you your loss i'm hope hear love man family god good friend prayers thoughts can hugs condolences bless strong brother

man i'm you shit made sad fuck fucking cry damn hear dude good sucks that's feels tear rip gonna words

amp you respects pay press sad post play alexa your comment bot questions removed our message stefan karl rules meme

you god our they your their his people life can church world them words believe him love when death faith

you your can yourself time feel life help don better things care make good people find hope when love try

you your work school job can time they good college people year make don't years working when help lot them

you it's don't your i'm you're people can they them that's can't feel when things time i've yourself make doesn't

Table 10: Labels and most probable words for condolence topics 
actually, you are overreact*, you overreacted, you're overreact* not that bad, as bad as, could be worse, *n't that bad,

at least (it|you|they|he|she), you shouldn't, I'm sorry you feel

Table 11: Phrases and regular expressions used to detect minimizing language, adapted from examples in Lehman et al. (1986) and Hogan et al. (1994)

\begin{tabular}{|c|c|c|}
\hline & rec. reply & rec. condolence \\
\hline hour1 & $-0.03^{* *}$ & -0.11 \\
\hline hour2 & $-0.04^{* *}$ & -0.13 \\
\hline hour3 & $-0.05^{* * *}$ & -0.06 \\
\hline hour4 & $-0.05^{* * *}$ & -0.05 \\
\hline hour5 & $-0.03^{*}$ & -0.11 \\
\hline hour6 & -0.03 & -0.12 \\
\hline hour7 & $0.03^{* *}$ & -0.11 \\
\hline hour8 & $0.05^{* * *}$ & -0.01 \\
\hline hour9 & $0.08^{* * *}$ & 0.07 \\
\hline hour 10 & $0.10^{* * *}$ & -0.06 \\
\hline hourl1 & $0.10^{* * *}$ & -0.08 \\
\hline hour 12 & $0.10^{* * *}$ & -0.07 \\
\hline hour13 & $0.10^{* * *}$ & 0.02 \\
\hline hour14 & $0.07^{* * *}$ & -0.02 \\
\hline hour15 & $0.03^{*}$ & -0.06 \\
\hline hour16 & $0.04^{* * *}$ & -0.06 \\
\hline hour17 & $0.03^{*}$ & -0.10 \\
\hline hour18 & 0.02 & -0.05 \\
\hline hour19 & 0.01 & -0.06 \\
\hline hour20 & $0.03^{* *}$ & -0.08 \\
\hline hour21 & 0.02 & 0.02 \\
\hline hour22 & $0.03^{* *}$ & 0.01 \\
\hline hour 23 & 0.02 & 0.02 \\
\hline month2 & 0.01 & $0.10^{* *}$ \\
\hline month3 & 0.02 & -0.04 \\
\hline month4 & 0.01 & $-0.08^{*}$ \\
\hline month5 & 0.01 & $-0.16^{* * *}$ \\
\hline month6 & 0.02 & $-0.08^{*}$ \\
\hline month7 & 0.01 & $-0.29^{* * *}$ \\
\hline month8 & 0.01 & $-0.11^{* *}$ \\
\hline month9 & 0.004 & $-0.09^{*}$ \\
\hline month10 & $-0.02^{* *}$ & $-0.20^{* * *}$ \\
\hline month11 & $-0.03^{* *}$ & $-0.19^{* * *}$ \\
\hline month 12 & $-0.04^{* * *}$ & $-0.26^{* * *}$ \\
\hline weekday1 & $0.02^{* *}$ & $0.07^{*}$ \\
\hline weekday2 & -0.01 & 0.05 \\
\hline weekday3 & 0.01 & -0.02 \\
\hline weekday4 & -0.01 & $0.13^{* * *}$ \\
\hline weekday5 & -0.01 & 0.01 \\
\hline weekday6 & 0.01 & $0.10^{* * *}$ \\
\hline $\log ($ length) & $0.29^{* * *}$ & $0.44^{* * *}$ \\
\hline depth & $0.09^{* * *}$ & $0.02^{* * *}$ \\
\hline score_post & $-0.0000^{* * *}$ & $0.0000^{*}$ \\
\hline time since post (hour) & $-0.01^{* * *}$ & $-0.01^{* * *}$ \\
\hline gender: female & -0.02 & $0.10^{* *}$ \\
\hline gender: male & 0.01 & -0.06 \\
\hline distress rating & $-0.08^{* * *}$ & $0.55^{* * *}$ \\
\hline topic: possessions & $0.16^{* * *}$ & -0.09 \\
\hline topic: politics & $0.69^{* * *}$ & 0.01 \\
\hline topic: moving & $0.24^{* * *}$ & -0.02 \\
\hline topic: dating & $0.23^{* * *}$ & $0.74^{* * *}$ \\
\hline topic: videogames & $0.24^{* * *}$ & $-0.50^{* * *}$ \\
\hline topic: medical & $0.38^{* * *}$ & $1.54^{* * *}$ \\
\hline topic: family & $0.10^{* * *}$ & $1.83^{* * *}$ \\
\hline topic: self reflection & $0.36^{* * *}$ & $0.72^{* * *}$ \\
\hline topic: videogames2 & $0.19^{* * *}$ & $-0.46^{* * *}$ \\
\hline topic: car accidents & $0.05^{* *}$ & $0.37^{* * *}$ \\
\hline topic: death & $0.18^{* * *}$ & $0.59^{* * *}$ \\
\hline topic: finances & $0.36^{* * *}$ & 0.17 \\
\hline topic: college & $0.39^{* * *}$ & $0.23^{* *}$ \\
\hline topic: sports & $0.23^{* * *}$ & $-0.43^{* * *}$ \\
\hline topic: depression & $0.40^{* * *}$ & $0.90^{* * *}$ \\
\hline topic: pets & $0.12^{* * *}$ & $1.59^{* * *}$ \\
\hline topic: diet & $0.33^{* * *}$ & -0.20 \\
\hline topic: advice & $0.26^{* * *}$ & $0.23^{* *}$ \\
\hline topic: death2 & $0.34^{* * *}$ & $0.50^{* * *}$ \\
\hline fear & $0.33^{* * *}$ & $1.53^{* * *}$ \\
\hline anger & $0.23^{* * *}$ & -0.55 \\
\hline trust & -0.08 & $-0.81^{* * *}$ \\
\hline surprise & $0.16^{* *}$ & $-1.12^{* * *}$ \\
\hline positive & $-0.24^{* * *}$ & -0.48 \\
\hline negative & -0.01 & 0.07 \\
\hline sadness & $0.22^{* * *}$ & $2.56^{* * *}$ \\
\hline disgust & $-0.27^{* * *}$ & -0.25 \\
\hline joy & $-0.35^{* * *}$ & $-1.37^{* * *}$ \\
\hline 1st person pronouns & $-0.01^{* * *}$ & $0.01^{* * *}$ \\
\hline 2nd person pronouns & $0.04^{* * *}$ & $-0.02^{* * *}$ \\
\hline 3rd person pronouns & $-0.01^{* * *}$ & $-0.004^{*}$ \\
\hline intercept & $-1.08^{* * *}$ & $-7.93^{* * *}$ \\
\hline Observations & $1,000,003$ & $1,000,003$ \\
\hline Log Likelihood & $-657,899.40$ & $-52,547.32$ \\
\hline Akaike Inf. Crit. & $1,315,961.00$ & $105,256.60$ \\
\hline Bayesian Inf. Crit. & $1,316,918.00$ & $106,213.70$ \\
\hline
\end{tabular}

Table 12: Full coefficients for the mixed-effect regression model of whether a distress message receives any reply (left) or a condolence (right). This is the expanded version of Table 2 in the main paper. See Table 10 for a description of topics. 


\begin{tabular}{|c|c|}
\hline $\begin{array}{l}\text { hour } 1 \\
\end{array}$ & -0.03 \\
\hline hour2 & -0.08 \\
\hline hour3 & $-0.11^{*}$ \\
\hline hour4 & $-0.16^{* *}$ \\
\hline hour5 & $-0.17^{* *}$ \\
\hline hour6 & -0.05 \\
\hline hour7 & -0.08 \\
\hline hour8 & \\
\hline hour9 & -0.04 \\
\hline hour10 & $-0.10^{* *}$ \\
\hline hour11 & -0.03 \\
\hline hour12 & 0.02 \\
\hline $\begin{array}{l}\text { hour13 } \\
\text { hes lo }\end{array}$ & 0.02 \\
\hline hour 14 & -0.05 \\
\hline hour 15 & -0.02 \\
\hline hour16 & -0.02 \\
\hline hour17 & 0.02 \\
\hline hour18 & 0.06 \\
\hline hour 19 & 0.003 \\
\hline hour20 & 0.02 \\
\hline hour21 & 0.07 \\
\hline hour22 & 0.04 \\
\hline hour23 & -0.01 \\
\hline month2 & -0.03 \\
\hline month3 & $-0.06^{*}$ \\
\hline month4 & -0.04 \\
\hline month5 & -0.02 \\
\hline month6 & -0.01 \\
\hline month7 & 0.004 \\
\hline month8 & $-0.10^{* * *}$ \\
\hline month9 & -0.004 \\
\hline month10 & -0.01 \\
\hline month11 & $-0.06^{*}$ \\
\hline month12 & 0.04 \\
\hline weekday 1 & 0.03 \\
\hline weekday 2 & -0.02 \\
\hline weekday3 & -0.02 \\
\hline weekday4 4 & 0.03 \\
\hline weekday5 5 & 0.001 \\
\hline weekday6 & 0.03 \\
\hline $\log ($ length) & $0.15^{* * *}$ \\
\hline $\begin{array}{l}\text { topic: estate/legal } \\
\text { tolitis }\end{array}$ & $-0.97^{* * *}$ \\
\hline topic: traveling & $-0.46^{* *}$ \\
\hline $\begin{array}{l}\text { topic: sports } \\
\text { tors }\end{array}$ & -0.19 \\
\hline topic: dieting & -0.12 \\
\hline topic: movies/song & $0.49^{* *}$ \\
\hline topic: video games & 0.03 \\
\hline topic: body parts & $-1.22^{* * *}$ \\
\hline topic: personal experience & $-0.55^{* *}$ \\
\hline topic: pets & $-1.16^{* * *}$ \\
\hline topic: shootings & -0.29 \\
\hline topic: medical & $-1.42^{* * *}$ \\
\hline topic: relationships & $-1.51^{* * *}$ \\
\hline topic: sympathy & $-0.91^{* * *}$ \\
\hline topic: cursing & $-0.77^{* * *}$ \\
\hline topic: memes & 0.21 \\
\hline topic: religion & $-1.00^{* * *}$ \\
\hline topic: advice & $-1.21^{* * *}$ \\
\hline topic: school & 0.20 \\
\hline topic: advice2 & 0.22 \\
\hline Post score & $-0.0000^{* * *}$ \\
\hline Condolence delay (min) & $-0.001^{* * *}$ \\
\hline Female author & 0.04 \\
\hline Male author & $-0.12^{* * *}$ \\
\hline Sentiment & $0.25^{* * *}$ \\
\hline Has adverbial "just"? & -0.01 \\
\hline Has minimization? & $-0.15^{* * *}$ \\
\hline (Buechel et al., 2018) empathy & $0.18^{* * * *}$ \\
\hline Appraisal-based empathy & $-0.17^{* * *}$ \\
\hline Lexical alignment & $0.37^{* * *}$ \\
\hline \# Third person pronouns & 0.001 \\
\hline \# Third person pronouns & $0.05^{* * *}$ \\
\hline \# Third person pronouns & $-0.05^{* * *}$ \\
\hline Constant & $-2.72^{* * *}$ \\
\hline Observations & 172,057 \\
\hline Log Likelihood & $-64,002.95$ \\
\hline Akaike Inf. Crit. & $128,155.90$ \\
\hline Bayesian Inf. Crit. & $128,910.10$ \\
\hline
\end{tabular}

Table 13: Full coefficients for the mixed-effect regression model of whether a condolence message receives a reply expressing gratitude. This is the expanded version of Table 5 in the main paper. 